\title{
THE TYPE OF CERTAIN BOREL SETS IN SEVERAL BANACH SPACES $\dagger$
}

\author{
BY \\ C. RAYMOND ADAMS AND JAMES A. CLARKSON
}

1. Introduction. Several writers have lately interested themselves in Borel sets in abstract spaces; especially in linear Borel sets in Banach spaces. In particular Mazur and Sternbach $\ddagger$ have shown that a linear $F_{\sigma \delta}$ need not be an $F_{\sigma}$. To prove this they resorted to a somewhat elaborate construction of sets having the desired properties instead of selecting a familiar subset of a space already much studied; and whether their examples are $G_{\delta \sigma}$ 's was not then determined. Shortly thereafter, however, Banach and Mazur\| gave two theorems concerning the Borel character of the convergence set (necessarily linear) of a sequence of linear operations, by aid of which they established that in every infinitely many-dimensional Banach space 9 there exists a linear $F_{\sigma \delta}$ which is not a $G_{\delta \sigma}$; and these theorems serve to fix completely the classification of some of the earlier examples.

Still more recently Oxtoby $\dagger \dagger$ has examined, in the Lebesgue spaces $\ddagger$ $L_{p}([0,1]),(p \geqq 1)$, the set $C$ of points corresponding to continuous functions and the set $R$ corresponding to (properly) Riemann integrable functions He showed that each of these sets is an $F_{\sigma \delta}$ of first category; but he left unanswered, for example, the question of whether they are $F_{\sigma}$ 's.

Following Oxtoby we consider here several familiar subsets of well known function spaces, and complete the determination of their Borel type. The sets

† Presented to the Society, September 6, 1938; received by the editors April 21, 1938.

$\ddagger$ Mazur and Sternbach, Über die Borelschen Typen von linearen Mengen, Studia Mathematica, vol. 4 (1933), pp. 48-53.

$\S$ Following Hausdorff we shall call a closed [open] set an $F$. [a $G$ ]; the sum [product] of countably many closed [open] sets an $F_{\sigma}\left[\mathrm{a} G_{\delta}\right.$ ]; the product [sum] of countably many $F_{\sigma}$ 's [ $G_{\delta}$ 's] an $F_{\sigma \delta}$ [a $G_{\delta \sigma}$ ]; and so on. $E$ is a set of order $\alpha(\geqq 0)$ in the $F$-classification [G-classification] if it is an $F$ [a $G$ ] with $\alpha$ subscripts. Since every $F[G]$ is a $G_{\delta}$ [an $F_{\sigma}$ ], any set of order $\alpha$ is ambiguous of order $\alpha+1$; that is, of order $\alpha+1$ in both classifications. A set of order $\alpha$ in both classifications, but of order $\alpha-1$ in neither, may be called properly ambiguous of order $\alpha$.

By definition, a set $E$ contained in a space $S$ is of first category in $S$ if it is the sum of countably many sets each non-dense in $S$; otherwise it is of second category in $S$.

|| Banach and Mazur, Eine Bemerkung über die Konvergenzmengen von Folgen linearer Operationen, Studia Mathematica, vol. 4 (1933), pp. 90-94.

If In a finitely many-dimensional Banach space a linear set is always closed.

$\dagger \dagger$ Oxtoby, The category and Borel class of certain subsets of $L_{p}$, Bulletin of the American Mathematical Society, vol. 43 (1937), pp. 245-248.

$\ddagger$ The notation $[a, b]$ will always designate the closed interval $a \leqq t \leqq b$. 
$C$ and $R \subset L_{p}$ will be shown to be unambiguous of order 2; the same will be proved for the set $R^{*} \subset L_{p}$ corresponding to functions each of which is properly or improperly Riemann integrable $\dagger$ over $[0,1]$, and for the set $A C$ of absolutely continuous functions in the space $C$ of continuous functions. The chief technical tool employed is Lemma 1, a characterization of $G_{\delta}$ sets in any metric space (complete or not) which seems easy to apply in many particular instances. One of the above mentioned theorems of Banach and Mazur can also be used. Because of the lack of established technique for dealing with questions of the sort considered here we feel that quite as much interest (if not more) may attach to our methods as to our results. Therefore we offer no apology for developing our argument in such form that some of the results are proved more than once; the course of reasoning we shall follow seems the more clearly to illustrate the methods employed and the more accurately to indicate their range and ease of application.

It may be of interest that in $L_{p}$ the set $C B V$ corresponding to continuous functions of bounded variation is properly ambiguous of order 2 .

We conclude this paper with a few remarks concerning other similar questions, including an illustrative application of our methods to a non-Banach space.

2. Preliminary theorems. Oxtoby has communicated to us the following theorem, of which he was in possession at the time his paper cited above was written; the simple proof given here, however, he obtained somewhat later.

THEOREM 1 (Oxtoby). $R^{*}$ is an $F_{\text {os }}$ of first category in each space $L_{p},(p \geqq 1)$.

Proof. If $x, x^{\prime}$ are arbitrary points of $L_{p}$ and $x(t), x^{\prime}(t)$ any functions representative of these points, we have $\ddagger\left|x_{N}(t)-x_{N}^{\prime}(t)\right| \leqq\left|x(t)-x^{\prime}(t)\right|$ for all $t$ in $[0,1]$ and all $N>0$; hence $T_{N}(x)=x_{N}$ is a continuous transformation of $L_{p}$ into a part of itself. Setting $R_{N}=T_{N}^{-1}(R)$, we may write $R^{*}=\prod_{N-1}^{\infty} R_{N}$. Each $R_{N}$, being the antecedent of an $F_{\sigma \delta}$ under $T_{N}$, is itself $\S$ an $F_{\sigma \delta}$; whence $R^{*}$ is an $F_{\sigma \delta}$. That $R^{*}$ is of first category follows at once $\|$ since it is a proper linear Borel subset of $L_{p}$.

THEOREM 2. $A C$ is an $F_{\text {os }}$ of first category in the space $C$.

$\dagger$ We shall regard a function $x(t) \varepsilon L_{1}$ unbounded on $[0,1]$ as improperly Riemann integrable if and only if for each $N>0$ the truncated function $x_{N}(t)$ defined as $-N, x(t)$, or $N$ according as $x(t)<-N,-N \leqq x(t) \leqq N$, or $N<x(t)$ is R-integrable and $\lim _{N \rightarrow \infty} \int_{0}^{1} x_{N}(t) d t$ exists. With this definition, $R^{*}$ is the set of points in $L_{p}$ corresponding to functions which are Lebesgue integrable and continuous almost everywhere.

$\ddagger$ Here $x_{N}(t)$ stands for the truncated function $x(t)$ as defined in footnote $\dagger$ of this issue.

\& See, for example, Kuratowski, Topologie I, Warsaw, 1933, p. 179.

|| See Banach, Thêorie des Opérations Linéaires, Warsaw, 1932, p. 36, Theorem 1. 
Proof. Let $E_{m n},(m, n=1,2,3, \cdots)$, represent the set of all continuous functions $x(t)$ such that for any set of nonoverlapping subintervals $t_{v}<t<t_{\nu}^{\prime}$ of $[0,1]$ with $\sum_{\nu}\left(t_{\nu}^{\prime}-t_{\nu}\right)<1 / m$ the condition $\sum_{\nu}\left|x\left(t_{\nu}^{\prime}\right)-x\left(t_{v}\right)\right| \leqq 1 / n$ is satisfied. For any fixed set of such subintervals, the condition imposed defines a closed set of points $x \in C$, and the product of any number of closed sets is closed; hence each $E_{m n}$ is closed. But we clearly have $A C=\prod_{n=1}^{\infty} \sum_{m=1}^{\infty} E_{m n}$. That $A C$ is of first category follows from Banach's theorem (loc. cit.).

3 . Concerning the $F_{\sigma}$ property. In this section we shall show that the sets mentioned in $\$ 1$ are not $F_{\sigma}$ 's in the spaces in question. We give first the following proof that the set $C$ is not an $F_{\sigma}$ in $L_{p}$.

It suffices to exhibit a set $S \subset C$ such that under every decomposition $S=\sum_{n=1}^{\infty} S_{n}$ at least one subset $S_{n}$ has a limit point in $L_{p}-C$. Let $C S$ designate the class of continuous singular functions, $\dagger D B V N$ the class of discontinuous functions of bounded variation having no external saltus. $\ddagger$ According to recent results of Adams and Morse, $\S$ if we introduce in the space $B V$ of functions of bounded variation the metric\|

$$
(x ; y)=\int_{0}^{1}|x(t)-y(t)| d t+\left|T_{0}{ }^{1}(x)-T_{0}{ }^{1}(y)\right|,
$$

$C S$ is a set of second category in $C S+D B V N$. Since $D B V N$ is dense in the sum set, $C S$ is not an $F_{\sigma}$ therein; that is to say, under every decomposition $C S=\sum_{n=1}^{\infty} H_{n}$ at least one set $H_{n}$ has a limit point in $D B V N$. Now convergence in the metric of $B V$ implies convergence in the metric of $L_{1}$, as well as uniform boundedness of the sequence of functions involved. Hence it follows that if $S$ is the set of points in $L_{p}$ corresponding to continuous singular functions and $S=\sum_{n=1}^{\infty} S_{n}$ is any decomposition whatever, at least one subset $S_{n}$ will have (in the metric of $L_{p}$ for all $p \geqq 1$ ) a limit point in the set of points of $L_{p}$ corresponding to $D B V N$. But a discontinuous function of bounded variation having no external saltus is not equivalent (in the metric of $L_{p}$ ) to a continuous function; consequently the subset $S_{n}$ in question has a limit point in $L_{p}-C$.

It may be observed that this reasoning shows that any set $E$ such that $C S \subset E \subset C \subset L_{p}$ is no $F_{\sigma}$ in $L_{p}$. Moreover a precisely similar argument, in

† A singular function is a function of bounded variation whose derivative vanishes almost everywhere; see, for example, Saks, Théorie de l'Intégrale, Warsaw, 1933, pp. $11 \mathrm{ff}$.

$\ddagger$ A function $x(t)$ of bounded variation is said to have no external saltus if for every $t_{1},\left(0 \leqq t_{1} \leqq 1\right)$, we have $\lim \inf _{t \rightarrow t_{1}} x(t) \leqq x\left(t_{1}\right) \leqq \lim \sup _{t \rightarrow t_{1}} x(t)$.

$\S$ Adams and Morse, On the space (BV), these Transactions, vol. 42 (1937), pp. 194-205; see especially the concluding paragraph of $\$ 3$.

$\|$ Throughout this paper the distance between two points $x, y$ in a metric space will be denoted by $(x, y)$. Here $T_{0}^{1}(z)$ stands for the total variation of $z(t)$ on the interval $0 \leqq t \leqq 1$. 
which the space $B V$ is metrized with the distance function $\dagger$

$$
(x, y)=\int_{0}^{1}|x(t)-y(t)| d t+\left|L_{0}^{1}(x)-L_{0}^{1}(y)\right|
$$

and $C S$ is replaced by $A C$, can be employed to establish that any set $E$ such that $A C \subset E \subset C \subset L_{p}$ is no $F_{\sigma}$ in $L_{p}$.

This type of proof, however, seems to be inapplicable to the other questions with which we are concerned. A method of attack with a much wider range of applicability is provided by the following lemma, which characterizes, by a property of the set itself, any $G_{\delta}$ in a metric space.

Lemma $\ddagger 1$. Let $E$ be a subset of a metric space $S$. A necessary and sufficient condition that $E$ be $a G_{\delta}$ in $S$ is the existence of a sequence of positive functions $\Delta_{n}(x),(n=1,2,3, \cdots)$, defined on $E$ and having the property that no sequence $\left\{x_{n}\right\} \subset E$ with $\left(x_{n}, x_{n+1}\right)<\Delta_{n}\left(x_{n}\right)$ for all $n$ converges to a point of $S-E$.

Proof. We consider first the necessity, and prove that if $E$ is a $G_{\delta}$, there exists a sequence of functions $\Delta_{n}(x)$ with the property asserted; in fact, each function $\Delta_{n}(x)$ exhibited will in addition satisfy a Lipschitz condition of order 1 on $E$. Let $E=\prod_{i-1}^{\infty} O_{i}$, where $O_{i}$ is open and $O_{i+1} \subset O_{i}$ for each $i$; let $\rho\left(x, \widetilde{O}_{i}\right),(x \in E ; i=1,2,3, \cdots)$, stand for the distance from $x$ to the set $\widetilde{O}_{i}=S-O_{i}$; and set $\Delta_{n}(x)=2^{-2 n} \rho\left(x, \widetilde{O}_{n}\right)$. If $\left\{x_{n}\right\} \subset E$ satisfies the condition $\left(x_{n}, x_{n+1}\right)<\Delta_{n}\left(x_{n}\right)$ for each $n$, we have for all $s$

$$
\Delta_{s+1}\left(x_{s+1}\right) \leqq 2^{-2 s-2}\left[\left(x_{s+1}, x_{s}\right)+\rho\left(x_{s}, \tilde{O}_{s+1}\right)\right]<2^{-2 s-1} \rho\left(x_{s}, \tilde{O}_{s}\right)=\Delta_{s}\left(x_{s}\right) / 2 .
$$

Hence for $k$ fixed and $n>k$ we infer

$$
\rho\left(x_{n}, \tilde{O}_{k}\right) \geqq \rho\left(x_{k}, \tilde{O}_{k}\right)-\sum_{s=k}^{n-1}\left(x_{s}, x_{s+1}\right)>\rho\left(x_{k}, \tilde{O}_{k}\right)-2 \Delta_{k}\left(x_{k}\right) \geqq \rho\left(x_{k}, \widetilde{O}_{k}\right) / 2 .
$$

Therefore $x_{n}$ cannot converge to a point of $\widetilde{O}_{k}$ for any $k$; hence it can converge to no point of $\sum_{k=1}^{\infty} \widetilde{O}_{k}=S-E$.

For the sufficiency, we note first that if there exists a sequence of functions $\Delta_{n}(x)$ with the property specified, there certainly will exist one which has the additional properties $\Delta_{n+1}(x) \leqq \Delta_{n}(x)<1 / n$ for all $x \& E$, all $n$. Assuming then the existence of a sequence $\Delta_{n}(x)$ with all these properties, we may set $E_{n}=\sum_{x \varepsilon E} K\left(x, \Delta_{n}(x)\right)$, where $K\left(x, \Delta_{n}(x)\right)$ stands for the open sphere of $S$ with center $x \in E$ and radius $\Delta_{n}(x)$. Then each $E_{n}$ is clearly open and $E \subset \prod_{n=1}^{\infty} E_{n}$. We shall show $E \supset \prod_{n=1}^{\infty} E_{n}$; whence $E=\prod_{n=1}^{\infty} E_{n}$ is a $G_{\delta}$. Let

$\dagger$ Here $L_{0}{ }^{1}(z)$ designates the (Peano) length of $z(t)$ on the interval $0 \leqq t \leqq 1$. The set $A C$ is of second category in $A C+D B V N$; see Adams and Morse, loc. cit., the last paragraph of p. 204.

$\ddagger$ This lemma, as well as its proof, is due entirely to Dr. Clarkson.-C.R.A. 
$z \varepsilon \prod_{n=1}^{\infty} E_{n}$; then $z \varepsilon E_{1}$ and there exists $x_{1} \varepsilon E$ such that $\left(z, x_{1}\right)<\Delta_{1}\left(x_{1}\right)$. Moreover, for every $n$ there exists a point $x_{n}^{\prime} \& E$ with $\left(z, x_{n}^{\prime}\right)<\Delta_{n}\left(x_{n}^{\prime}\right)<1 / n$; the sequence $\left\{x_{n}^{\prime}\right\}$ tends to $z$, and for $n$ sufficiently large we have $\left(x_{1}, x_{n}^{\prime}\right)<\Delta_{1}\left(x_{1}\right)$. Let $x_{n}^{\prime}$ (with $n$ sufficiently large, and greater than or equal to 2 ) be taken as $x_{2}$; then we have $\left(z, x_{2}\right)=\left(z, x_{n}^{\prime}\right)<\Delta_{n}\left(x_{n}^{\prime}\right)=\Delta_{n}\left(x_{2}\right) \leqq \Delta_{2}\left(x_{2}\right)$. Next choose from $\left\{x_{n}^{\prime}\right\}$ an element such that $\left(x_{2}, x_{n}^{\prime}\right)<\Delta_{2}\left(x_{2}\right)$ with $n \geqq 3$ and call it $x_{3}$. Continuing indefinitely this process of selection we obtain $\left\{x_{n}\right\}$, a subsequence of $\left\{x_{n}^{\prime}\right\}$, with $\left(x_{n}, x_{n+1}\right)<\Delta_{n}\left(x_{n}\right)$ for every $n$ and $x_{n} \rightarrow z$. The property of $\Delta_{n}(x)$ stated in the lemma being assumed, we have $z \& E$.

THEOREM 3. The sets $C, R$, and $R^{*}$ in each space $L_{p},(p \geqq 1)$, and the set $A C$ in the space $C$, are no $F_{\sigma}$ 's.

Proof. We begin with a second proof that $C$ is not an $F_{\sigma}$ in $L_{p}$, using Lemma 1 to show that $L_{p}-C$ is not a $G_{\delta}$. The basic idea of that lemma is that a sequence of points in a $G_{\delta}$ which converges rapidly enough cannot tend to a limit outside the $G_{\delta}$, the rapidity of the convergence being prescribed by the condition $\left(x_{n}, x_{n+1}\right)<\Delta_{n}\left(x_{n}\right)$. The following argument shows that, given any sequence whatsoever of positive functions $\Delta_{n}(x)$ defined on $L_{p}-C$, there always exists a sequence $\left\{x_{n}\right\} \subset L_{p}-C$, converging to a point in $C$, with $\left\|x_{n}-x_{n+1}\right\|<\Delta_{n}\left(x_{n}\right)$ for every $n$.

Let $p$ be fixed, let $\left\{t_{n}\right\}$ be any sequence satisfying the conditions $1 / 2=t_{1}<t_{2}<\cdots<t_{n}<\cdots, t_{n} \rightarrow t_{0} \leqq 1$, and define $x_{1}(t)$ as the characteristic function of the interval $\left[0, t_{1}\right]$. In general, $x_{n}(t)$ having been defined, let $x_{n+1}(t)=x_{n}(t)$ for $t$ not in the interval $\left[t_{n}, t_{n+1}\right]$; in that interval let $x_{n+1}(t)$ be linear, with $x_{n+1}\left(t_{i}\right)=2^{1-i},(i=n, n+1)$. That each $x_{n} \varepsilon L_{p}-C$ and that $x_{n} \rightarrow x_{\varepsilon} C$ is apparent. Moreover, it is clear that $t_{1}, t_{2}, \cdots, t_{n}$ having been fixed, we have $\lim _{t_{n+1} \rightarrow t_{n}}\left\|x_{n}-x_{n+1}\right\|=0$, so that by a proper selection of the sequence $\left\{t_{n}\right\}$ the condition $\left\|x_{n}-x_{n+1}\right\|<\Delta_{n}\left(x_{n}\right)$ may be satisfied for every $n$. This completes the proof for $C \subset L_{p}$. Actually $x \in A C$, so we have also proved that $A C$ is no $F_{\sigma}$ in $L_{p}$.

By a precisely similar argument we may dispose of $R$ and $R^{*}$ together, showing that for any sequence of positive functions $\Delta_{n}(x)$ defined on $L_{p}-R^{*}$ there exists a sequence $\left\{x_{n}\right\} \subset L_{p}-R^{*}$, with $x_{n} \rightarrow x \& R$ and $\left\|x_{n}-x_{n+1}\right\|<\Delta_{n}\left(x_{n}\right)$ for each $n$. Let $y(t)$ be a function in the class $\dagger L_{p}$, with $|y(t)|<1$ for all $t$, which in no interval $\left[2^{-n-1}, 2^{-n}\right]$ is equivalent to a function in class $R^{*}$. We define $x_{1}(t)=0$ for $0 \leqq t \leqq 1 / 2, x_{1}(t)=y(t)$ otherwise; and in general, $x_{n}(t)$ having been defined, set $x_{n+1}(t)=0$ for $0 \leqq t<2^{-n-1}, x_{n+1}(t)=k_{n+1} y(t)$ for $2^{-n-1} \leqq t<2^{-n}$, define $x_{n+1}(t)$ as a continuous function in absolute value always less than 1 approximating $x_{n}(t)$ in the norm of $L_{p}$ for $2^{-n} \leqq t<2^{-n+1}$, and set

† We employ the term class $L_{p}$ when an element is to be thought of as a single function. 
$x_{n+1}(t)=x_{n}(t)$ for $2^{-n+1} \leqq t \leqq 1$, where $\left\{k_{n}\right\}$ is a sequence of numbers with $0<k_{n}<1$ for each $n$. This time it is clear that every $x_{n} \varepsilon L_{p}-R^{*}$; and $x_{n} \rightarrow x \varepsilon R$, since $x(t)$ is bounded and continuous almost everywhere. At each step of the process of definition the condition $\left\|x_{n}-x_{n+1}\right\|<\Delta_{n}\left(x_{n}\right)$ will be satisfied if we merely choose the constant $k_{n+1}$ sufficiently small and the continuous function in question to approximate $x_{n}(t)$ closely enough in the norm of $L_{p}$.

That $A C$ is no $F_{\sigma}$ in space $C$ may be established by a proof that so closely follows the line of the above argument that we leave its details to the reader, remarking only that the construction may conveniently be based on functions of the Cantor ternary type.

4. Concerning the $G_{\delta \sigma}$ property. Our main object here is to establish the following theorem.

Theorem 4. The sets $C, R$, and $R^{*}$ in each space $L_{p},(p \geqq 1)$, and the set $A C$ in the space $C$, are no $G_{\delta \sigma}$ 's.

For $A C$ in space $C$ a proof may be constructed by aid of this lemma:

LEMMa 2. Let $E$ be a subset of the space $\dagger A C$ which is dense in some sphere of $A C$; then $E$ contains a sequence which converges with arbitrary rapidity (in the sense specified by Lemma 1 ) in the metric of $C$ to a point in $C-A C$.

Proof of the lemma. On account of the homogeneity of $A C$ as a Banach space, it suffices to consider the case in which $E$ is dense in $K$, the unit sphere about the zero element as center. Let $\Delta_{n}(x)$ be any sequence whatever of positive functions defined on $E$. Then $x_{1}(t)$ may be taken as any element of $E \cdot K$ satisfying the inequality $1 / 2<T_{0}^{1}\left(x_{1}\right)<1$. It is apparent that there exists a polygonal function $p_{1}(t) \varepsilon K$, its graph consisting of segments alternately horizontal and otherwise, such that the norm in $C,\left\|x_{1}-p_{1}\right\|_{c}$, is less than $\Delta_{1}\left(x_{1}\right) / 2 ; T_{0}{ }^{1}\left(p_{1}\right)$ exceeds $1 / 2$; and the sum of the projections on the $t$-axis of the segments of the graph that are not horizontal, say $\sum_{v-1}^{m_{1}}\left(t_{2 \nu}^{(1)}-t_{2 \nu-1}^{(1)}\right)$, is less than $1 / 2$. By virtue of the density of $E$ in $K$, there exists a function $x_{2}(t) \& E \cdot K$ with $\left\|p_{1}-x_{2}\right\|_{c}<\Delta_{1}\left(x_{1}\right) / 2$ and

$$
\sum_{v=1}^{m_{1}}\left|x_{2}\left(t_{2 v}^{(1)}\right)-x_{2}\left(t_{2 v-1}^{(1)}\right)\right|=1 / 2+\delta_{1}, \quad \delta_{1}>0 .
$$

Next we choose a polygonal function $p_{2}(t) \varepsilon K$, its graph consisting of segments alternately horizontal and sloping, such that $\left\|x_{2}-p_{2}\right\|_{c}$ is less than $\min \left[\Delta_{2}\left(x_{2}\right) / 2, \delta_{1} /\left(8 m_{1}\right)\right] ; T_{0}{ }^{1}\left(p_{2}\right)$ exceeds $1 / 2 ;$ and the sum of the projections on the $t$-axis of the sloping segments of the graph, say $\sum_{\nu=1}^{m_{2}}\left(t_{2 \nu}^{(2)}-t_{2 \nu-1}^{(2)}\right)$, is

$\dagger$ The space $A C$, here understood to be normed with $\|x\|=|x(0)|+T_{0}{ }^{1}(x)$, is a Banach space. 
less than $1 / 2^{2}$. Then we select a function $x_{3}(t) \varepsilon E \cdot K$ with $\left\|p_{2}-x_{3}\right\|_{C}<$ $\min \left[\Delta_{2}\left(x_{2}\right) / 2, \delta_{1} /\left(8 m_{1}\right)\right]$ and

$$
\sum_{\nu=1}^{m_{2}}\left|x_{3}\left(t_{2 \nu}^{(2)}\right)-x_{3}\left(t_{2 \nu-1}^{(2)}\right)\right|=1 / 2+\delta_{2}, \quad \delta_{2}>0 .
$$

In the third stage of this procedure $p_{3}(t) \varepsilon K$ would be chosen to satisfy the inequalities

$$
\begin{gathered}
\left\|x_{3}-p_{3}\right\|_{C}<\min \left[\Delta_{3}\left(x_{3}\right) / 2, \delta_{1} /\left(16 m_{1}\right), \delta_{2} /\left(8 m_{2}\right)\right], T_{0}{ }^{1}\left(p_{3}\right)>1 / 2, \\
\sum_{v=1}^{m_{3}}\left(t_{2 v}^{(3)}-t_{2 v-1}^{(3)}\right)<1 / 2^{3} ;
\end{gathered}
$$

and $x_{4}(t) \varepsilon E \cdot K$ to fulfill the conditions

$$
\begin{gathered}
\left\|p_{3}-x_{4}\right\|_{C}<\min \left[\Delta_{3}\left(x_{3}\right) / 2, \delta_{1} /\left(16 m_{1}\right), \delta_{2} /\left(8 m_{2}\right)\right], \\
\sum_{\nu=1}^{m_{3}}\left|x_{4}\left(t_{2 \nu}^{(3)}\right)-x_{4}\left(t_{2 v-1}^{(3)}\right)\right|=1 / 2+\delta_{3},
\end{gathered}
$$

And so we continue for subsequent stages, indefinitely. It is quite clear that $\left\{x_{n}\right\}$ is a Cauchy sequence in the metric of space $C$, and that if $x$ is its limit, we have $\sum_{\nu=1}^{m_{k}}\left|x\left(t_{2 \nu}^{(\boldsymbol{k})}\right)-x\left(t_{2 \nu-1}^{(\boldsymbol{k})}\right)\right|>1 / 2$ for each integer $k \geqq 1$; since $\sum_{\nu=1}^{m_{k}}\left(t_{2 \nu}^{(\mathbf{k})}-t_{2 \nu-1}^{(\mathbf{k})}\right) \rightarrow 0$ with $1 / k$, we have $x \varepsilon C-A C$, and the proof of the lemma is complete.

Returning now to the proof of Theorem 4 for $A C$ in space $C$, we observe that since the space $A C$ is complete and consequently of second category in itself, under any decomposition whatsoever $A C=\sum_{n=1}^{\infty} E_{n}$ at least one set $E_{n}$ must be dense (in the metric of $A C$ ) in some sphere of $A C$. By Lemma 2 we conclude that this set $E_{n}$ contains a sequence of the sort specified in the statement of that lemma. Hence, by Lemma $1, E_{n}$ is no $G_{\delta}$ in $C$; and $A C$ is no $G_{\delta \sigma}$.

For $C, R$, and $R^{*}$ in space $L_{p}$ one may employ an entirely similar proof using the following lemma.

LemMa 3. Let $E$ be a subset of the space $C$ which is dense in some sphere of $C$; then $E$ contains a sequence which converges with arbitrary rapidity (in the sense specified by Lemma 1$)$ in the metric of $L_{p},(p \geqq 1)$, to a point in $L_{p}-R^{*}$.

Proof of the lemma. On account of the homogeneity of $C$ as a Banach space, it is sufficient to consider the case in which $E$ is dense in $K$, the unit sphere about the zero element as center. Let $\Delta_{n}(x)$ be any sequence whatever of positive functions defined on $E$. A sequence of closed subintervals $\delta_{j}$, $(j=1,2,3, \cdots)$, of $I=[0,1]$ is to be chosen as follows: $\delta_{1}$ is concentric with $I ; \delta_{2}$ and $\delta_{3}$ are concentric with the two intervals to the left and right, respectively, of $\delta_{1}$; the next four $\delta_{j}$ are concentric, respectively, with the four 
subintervals of $I$ which constitute the point set $I-\left(\delta_{1}+\delta_{2}+\delta_{3}\right)$; and so on, so that the point set $\sum_{j=1}^{\infty} \delta_{j}$ is dense in $I$. For each $j$ we allow $\delta_{j}^{\prime}$ to stand for the closed middle third of $\delta_{j}$.

At the outset the condition $m\left(\sum_{j=1}^{\infty} \delta_{j}\right)<1$ will be imposed.

As a first step in the construction of the required sequence of functions $x_{n}(t)$, let $\delta_{1}$ be chosen arbitrarily and $x_{1}(t) \varepsilon E \cdot K$ so that

$$
x_{1}(t)>3 / 4 \text { for } t \varepsilon \delta_{1}^{\prime}, \quad x_{1}(t)<1 / 4 \text { for } t \varepsilon I-\delta_{1} .
$$

The density of $E$ in $K$ clearly insures the existence of such a function $x_{1}(t)$. Secondly, if $\delta_{2}$ and $\delta_{3}$ are small enough, there will exist a function $x_{2}(t) \varepsilon E \cdot K$ with

$x_{2}(t)>3 / 4$ for $t \varepsilon \delta_{1}^{\prime}+\delta_{2}^{\prime}+\delta_{3}^{\prime}, \quad x_{2}(t)<1 / 4$ for $t \varepsilon I-\left(\delta_{1}+\delta_{2}+\delta_{3}\right)$,

$$
\left(x_{1}, x_{2}\right)<\Delta_{1}\left(x_{1}\right),
$$

where $\left(x_{1}, x_{2}\right)=\left\|x_{1}-x_{2}\right\|$ in $L_{p}$; again this is insured by the density of $E$ in $K$, and we assume $x_{2}(t)$ to be so chosen. Let this procedure be continued indefinitely; at each stage the existence of sufficiently small intervals $\delta_{j}$, and of a suitable function $x_{n}(t)$ to satisfy the desired conditions (whose nature must now be apparent to the reader), should be clear from the steps already described in detail. The imposition of a further condition, such as, for example, $\left(x_{n}, x_{n+1}\right)<2^{-n}$, will insure that $x_{n}(t)$ converge in $L_{p}$ to some function $x(t)$.

For $t \varepsilon H=I-\sum_{j=1}^{\infty} \delta_{j}$, each function $x_{n}(t)$ is less than $1 / 4$, and $m(H)$ is greater than 0 ; hence $x(t) \leqq 1 / 4$ almost everywhere in $H$, and by aid of metric density we infer ess $\lim \inf _{t \rightarrow t_{1}} x(t) \leqq 1 / 4$ for almost all $t_{1} \varepsilon H$. On the other hand, for $t$ in any interval $\delta_{j}^{\prime}$, all $x_{n}(t)$ with $n$ sufficiently large are greater than $3 / 4$, whence $x(t) \geqq 3 / 4$ for almost all $t \varepsilon \sum_{j=1}^{\infty} \delta_{i}^{\prime}$; since each point of $H$ is a limit point of intervals $\delta_{j}{ }^{\prime}$, this implies ess lim sup ${ }_{t \rightarrow t_{1}} x(t) \geqq 3 / 4$ everywhere in $H$. Thus there is a set of measure greater than zero at each point of which the essential saltus of $x(t)$ is greater than or equal to $1 / 2$, and $x \in L_{p}-R^{*}$.

We may now show that any set $E$ such that $C \subset E \subset R^{*} \subset L_{p}$ is no $G_{\delta \sigma}$ in $L_{p}$. Since space $C$ is of second category in itself, under any decomposition whatever $E=\sum_{n=1}^{\infty} E_{n}$ at least one of the sets $C \cdot E_{n}$ contains a set of continuous functions which is dense (in the metric of $C$ ) in some sphere of $C$. The conclusion follows from Lemmas 3 and 1.

Oxtoby (loc. cit.) has proved that the set $S_{u}\left[S_{l}\right] \subset L_{p}$ of points corresponding to functions each of which is upper [lower] semicontinuous on $[0,1]$ is an $F_{\sigma \delta}$, and has observed that $R=S_{u} \cdot S_{l}$. Since $S_{u}$ and $S_{l}$ are clearly of the same Borel type, the determination of their type is made precise by the following corollary. 
CoRollary. The sets $S_{u}$ and $S_{l}$ in each space $L_{p},(p \geqq 1)$, are no $G_{\delta \sigma}$ 's.

For $A C$ in space $C$, and $C$ in space $L_{p}$, alternative proofs $\dagger$ of Theorem 4 can easily be made by means of Theorem 3 and the following result already spoken of in $\$ 1$.

LEMma 4 (Banach and Mazur). Let $U_{n}(x),(n=1,2,3, \cdots)$, be a sequence of operations each linear $\ddagger$ on a Banach space $S$ to a like space $S^{\prime}$, and let $E$ be the subset of $S$ at each point $x$ of which $U_{n}(x)$ converges in $S^{\prime}$. If $E$ is a $G_{\delta \sigma}$, it is an $F_{\sigma}$.

A sequence of linear operations on space $C$ to $L_{1}$ having $A C$ for convergence set is provided by the following lemma (we choose $U_{n}(x)=p_{n}^{\prime}(t)$ ).

Lemma 5. Let $x \in C$ and let $p_{n}(t)$ be the polygonal function inscribed in $x(t)$ with "corners" at $t=m / n,(n=1,2,3, \cdots ; m=0,1, \cdots, n)$. A necessary and sufficient condition that $x \& A C$ is that the sequence $p_{n}^{\prime}(t)$ converge in the space $L_{1}$.

Proof of the lemma. For the necessity we have $x \in A C$ and $p_{n}(t)$ converging in length to $x(t)$; by a theorem of Adams and Lewy $\S$ it follows that $T_{0}{ }^{1}\left(p_{n}-x\right) \rightarrow 0$. But since $p_{n} \varepsilon A C$ for each $n$, we have

$$
T_{0}^{1}\left(p_{n}-x\right)=\int_{0}^{1}\left|p_{n}^{\prime}(t)-x^{\prime}(t)\right| d t .
$$

For the sufficiency we may assume without real restriction that $x(0)=0$. Then, if $p_{n}^{\prime}(t) \rightarrow y(t) \varepsilon L_{1}$, we may set $z(t)=\int_{0}^{t} y(s) d s$ and obtain for all $t$ in $[0,1]$

$$
\left|p_{n}(t)-z(t)\right|=\left|\int_{0}^{1}\left[p_{n}^{\prime}(s)-y(s)\right] d s\right| \leqq \int_{0}^{1}\left|p_{n}^{\prime}(s)-y(s)\right| d s \rightarrow 0 .
$$

Since also $\left|p_{n}(t)-x(t)\right| \rightarrow 0$ for all $t$, we infer $x(t) \equiv z(t)$.

For $C \subset L_{p}$ a similar proof can be constructed. Let us consider first the case of $p=1$, and for each $x \varepsilon L_{1}$, each integer $n \geqq 1$, define

$$
U_{n}(x)=y_{n}(\xi)=\left\{\begin{array}{l}
n \int_{\xi}^{\xi+1 / n} x(t) d t \text { for } 0 \leqq \xi \leqq 1-1 / n, \\
y_{n}(1-1 / n) \text { for } 1-1 / n \leqq \xi \leqq 1 .
\end{array}\right.
$$

$\dagger$ The applicability of these alternative proofs, however, is limited to Banach spaces. Moreover one such proof cannot yield the desired result for an entire range of sets in a space, as does, for example, the above proof based on Lemma 3.

$\ddagger$ Linear in the sense of Banach; that is, additive and continuous.

8 Adams and Lewy, On convergence in length, Duke Mathematical Journal, vol. 1 (1935), pp. 1926, Theorem 4. 
Then every $U_{n}(x)$ is a linear operation on $L_{1}$ to space $C$. If $x \varepsilon C \subset L_{1}$, and $\xi$ is any point in the interval $0 \leqq \xi<1$, there exists $\xi^{\prime}$ with $0 \leqq \xi^{\prime}-\xi \leqq 1 / n$ and $y_{n}(\xi)=x\left(\xi^{\prime}\right)$, so that the uniform continuity of a representative function $x(t)$ implies $y_{n}(t) \rightarrow x(t)$ uniformly on $[0,1]$. On the other hand, if the sequence $y_{n}(\xi)$ converges in space $C$, let $y(\xi)$ be its limit; that $y(\xi)=x(\xi)$ for almost all $\xi$ is well known, and we have $y \varepsilon C$. Hence $C \subset L_{1}$ is the convergence set of the sequence of linear operations $U_{n}(x)$. For the case of $p>1$ the same reasoning is valid, $\uparrow$ the linearity of each operation being a consequence of the inequality $\left\|U_{n}(x)\right\| \leqq n^{1 / p}\|x\|$.

Success has not attended our efforts to find a sequence of linear operations on $L_{p}$ having for its convergence set either $R$ or $R^{*}$.

We conclude this section by exhibiting a properly ambiguous set of order 2 in $L_{p}$; namely, $C B V$. That is, we shall establish the following theorem.

Theorem 5. The set $C B V$ in each space $L_{p},(p \geqq 1)$, is simultaneously an $F_{\sigma \delta}$ and $a G_{\delta \sigma}$, without being either an $F_{\sigma}$ or $a G_{\delta}$.

To prove this theorem we note first that $B V \subset L_{p}$ may easily be proved $\ddagger$ an $F_{\sigma}$ by setting $B V=\sum_{n-1}^{\infty} E_{n}$ where $E_{n}$ is the set of points each of which has a representative function $x(t)$ with $T_{0}{ }^{1}(x) \leqq n$. That each $E_{n}$ is closed in $L_{p}$ is an immediate consequence of a well known theorem of Helly. $\S$

Now it is easily seen that in $L_{p}, C B V=C \cdot B V$. Hence, in the light of Oxtoby's result (loc. cit.) that $C$ is an $F_{\sigma \delta}, C B V$ is an $F_{\sigma \delta}$; that it is no $F_{\sigma}$ has been shown above in $\S 3$, and that it is no $G_{\delta}$ follows from the theorem of Mazur and Sternbach just cited. $\ddagger$ It therefore remains only for us to prove that $C B V$ is a $G_{\delta \sigma}$. This will be done by aid of the following lemma.

Lemma 6. Let $x(t), x_{0}(t)$ be elements of the class $C B V$, and let $\left\|x-x_{0}\right\|_{C}$ and $\left\|x-x_{0}\right\|_{L_{p}}$ represent the norms in the spaces $C$ and $L_{p},(p \geqq 1)$, respectively; then the relation $\left\|x-x_{0}\right\|_{L_{p} \rightarrow 0}$ implies

$$
\lim \inf \left(T_{0}{ }^{1}(x)-\left\|x-x_{0}\right\|_{c}\right) \geqq T_{0}{ }^{1}\left(x_{0}\right) .
$$

Proof of the lemma. It suffices to establish the conclusion for the case of a

$\dagger$ Another proof for $C \subset L_{p}$ can be based on the following simple lemma, which provides a sequence of linear operations $\left(U_{n}(x)=s_{n}(t)\right)$ on $L_{p}$ to space $M$ (or $L_{\infty}$ ) having $C$ for convergence set. Let $x$ be an arbitrary element of $L_{p}$, and let $s_{n}(t)$ be the step-function defined on each subinterval $m / n \leqq t<(m+1) / n,(n=1,2,3, \cdots ; m=0,1, \cdots, n-1)$, as the integral mean of $x(t)$ on that subinterval, $s_{n}(1)=s_{n}(1-0)$, so that $s_{n}(t)$ is an element of the class $M$ (or $\left.L_{\infty}\right)$ of essentially bounded measurable functions. Then a necessary and sufficient condition that $x \& C$ is that the sequence $s_{n}$ converge in

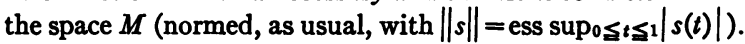

$\ddagger$ Mazur and Sternbach (loc. cit.) have proved that a linear $G_{\delta}$ in a Banach space is closed; hence $B V$ is no $G_{\delta}$, and its Borel type is completely determined.

$\S$ Helly, Über lineare Funktionaloperationen, Sitzungsberichte der Wiener Akademie, class IIa, vol. 121 (1912), p. 283. 
sequence $x_{n}(t),(n=1,2,3, \cdots)$, with $\left\|x_{n}-x_{0}\right\|_{L_{p} \rightarrow 0 \text {. This result will follow }}$ at once if we show that $x_{n}(t) \rightarrow x_{0}(t)$ on a set $D$ of points $t$ dense in $[0,1]$ implies the conclusion.

Let $\epsilon$ be an arbitrary positive number and let $S: 0=t_{0}<t_{1}<t_{2}<\cdots<t_{k}=1$ be a set of points with $t_{i} \varepsilon D$ for $i=1,2, \cdots, k-1$ and such that

$$
T_{t_{i-1}}^{t_{i}}\left(x_{0}\right)<\epsilon \text { for } i=1,2, \cdots, k ; \quad \sum_{i=1}^{k}\left|x_{0}\left(t_{i}\right)-x_{0}\left(t_{i-1}\right)\right|>T_{0}^{1}\left(x_{0}\right)-\epsilon .
$$

Next let $N$ be such that for all $n>N$ we have

$$
\left|x_{n}\left(t_{i}\right)-x_{0}\left(t_{i}\right)\right|<\epsilon /(2 k), \quad i=1,2, \cdots, k-1 ;
$$

whence

$$
\sum_{i=2}^{k-1}\left|x_{n}\left(t_{i}\right)-x_{n}\left(t_{i-1}\right)\right|>\sum_{i=2}^{k-1}\left|x_{0}\left(t_{i}\right)-x_{0}\left(t_{i-1}\right)\right|-\epsilon>T_{0}{ }^{1}\left(x_{0}\right)-4 \epsilon .
$$

We now fix $n$ as any integer greater than $N$. Let $t^{\prime}$ be a point where

$$
\max _{0 \leqq t \leqq 1}\left|x_{n}(t)-x_{0}(t)\right|
$$

is assumed. Then $t^{\prime}$ is contained in one of the subintervals determined by $S$, say in $\left[t_{l-1}, t_{l}\right]$; and for at least one of the end points of this subinterval, say $t_{l}$, inequality (1) holds. Therefore we have

$$
\begin{aligned}
\left|x_{n}\left(t^{\prime}\right)-x_{n}\left(t_{l}\right)\right| & \geqq\left|x_{n}\left(t^{\prime}\right)-x_{0}\left(t^{\prime}\right)\right|-\left|x_{0}\left(t^{\prime}\right)-x_{0}\left(t_{l}\right)+x_{0}\left(t_{l}\right)-x_{n}\left(t_{l}\right)\right| \\
& \geqq\left\|x_{n}-x_{0}\right\|_{c}-2 \epsilon ;
\end{aligned}
$$

whence

$$
\begin{aligned}
T_{0}^{1}\left(x_{n}\right) & \geqq\left[\sum_{i=2}^{l-1}+\sum_{i=l+1}^{k-1}\right]\left|x_{n}\left(t_{i}\right)-x_{n}\left(t_{i-1}\right)\right|+\left|x_{n}\left(t^{\prime}\right)-x_{n}\left(t_{l}\right)\right| \\
& \geqq\left[\sum_{i=2}^{l-1}+\sum_{i=l+1}^{k-1}\right]\left|x_{0}\left(t_{i}\right)-x_{0}\left(t_{i-1}\right)\right|-\epsilon+\left\|x_{n}-x_{0}\right\|_{c}-2 \epsilon \\
& \geqq \sum_{i=2}^{k-1}\left|x_{0}\left(t_{i}\right)-x_{0}\left(t_{i-1}\right)\right|+\left\|x_{n}-x_{0}\right\|_{C}-4 \epsilon \\
& \geqq T_{0}^{1}\left(x_{0}\right)+\left\|x_{n}-x_{0}\right\|_{c}-7 \epsilon,
\end{aligned}
$$

and the proof of the lemma is complete.

Returning now to the proof of Theorem 5, we set $C B V=\sum_{k=1}^{\infty} E_{k}$ where $E_{k}$ is the set of points in $C B V \subset L_{p}$ each of which has a representative function $x(t)$ with $T_{0}^{1}(x) \leqq k$. It suffices to show that each $E_{k}$ is a $G_{\delta}$ in $L_{p}$. Having fixed $k$, we define the function $\Delta_{n}(x)$ specified in Lemma 1 as follows: for 
each $x \varepsilon E_{k}$, each integer $n \geqq 1$, let $\Delta_{n}(x)=r>0$ where $r$ is such that for $y \varepsilon E_{k} \cdot K(x, r)$ we have

$$
\|x-y\|_{C} \leqq T_{0}^{1}(y)-T_{0}^{1}(x)+1 / 2^{n},
$$

whence

$$
T_{0}^{1}(y)-T_{0}^{1}(x) \geqq-1 / 2^{n},
$$

this $r$ existing in consequence of Lemma 6. If $x_{n},(n=1,2,3, \cdots)$, is any

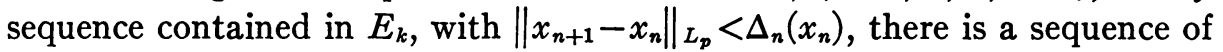
representative functions $x_{n}(t)$ for which we have

$$
\sum_{n=1}^{\infty}\left\|x_{n+1}-x_{n}\right\|_{C} \leqq \sum_{n=1}^{\infty}\left[T_{0}^{1}\left(x_{n+1}\right)-T_{0}{ }^{1}\left(x_{n}\right)\right]+\sum_{n=1}^{\infty} 1 / 2^{n} .
$$

The first series on the right is easily proved convergent, since its partial sum $T_{0}{ }^{1}\left(x_{m}\right)-T_{0}{ }^{1}\left(x_{1}\right)$ is "almost non-decreasing" by virtue of inequality (2) and is bounded from above by $2 k$. Hence $x_{n}(t)$ converges uniformly, say to $x(t) \varepsilon C ; T_{0}{ }^{1}(x) \leqq k$ by Lemma 6 ; the corresponding element $x \in L_{p}$ to which $x_{n}$ converges in $L_{p}$ is in $\bar{E}_{k}$; and $E_{k}$ is a $G_{\delta}$ in $L_{p}$ by Lemma 1.

5. Other questions. The above sections afford methods and illustrate techniques by means of which certain questions concerning the Borel character of many sets in various metric spaces can be settled. Without prolonging our discussion unduly we may list here, with slight indications of proof, the answers to a few questions which the reader might very naturally raise in view of the sets and spaces already considered.

I. $A C$ in the spaces $M$ and $L_{p}$ is an $F_{\sigma \delta}, n o G_{\delta \sigma}$.

II. $C B V$ in the spaces $C$ and $M$ is an $F_{\sigma}, n o G_{\delta}$.

III. $B V$ in the space $M$ is an $F_{\sigma}$, no $G_{\delta}$.

IV. $C$ in the space $M$ is an $F$, no $G$.

V. $R$ in the space $M$ is an $F$, no $G$.

In each case the set is of first category in the space in question, according to the theorem of Banach cited earlier. In cases I-III the set is no $G_{\delta}$ by virtue of the theorem of Mazur and Sternbach cited in $\$ 4$. For determining the $F$-classification of $A C$ in $L_{p}$ the following observations may be helpful.

(i) Let $x_{n}(t),(n=1,2,3, \cdots)$, be continuous on $[0,1]$ and $x_{n}(t) \rightarrow x(t)$ in the norm of $L_{p}$. If for arbitrary $\epsilon>0$ there exists $\delta>0$ such that for every $n$ we have

$$
\left|x_{n}\left(t^{\prime}\right)-x_{n}\left(t^{\prime \prime}\right)\right|<\epsilon \text { for }\left|t^{\prime}-t^{\prime \prime}\right|<\delta,
$$

then this condition is satisfied also by $x(t)$.

(ii) For fixed $m, n,(m, n=1,2,3, \cdots)$, the functions $x(t)$, continuous on 
$[0,1]$ and such that for any set of nonoverlapping intervals $t_{\nu}<t<t_{\nu}^{\prime}$ with $\sum_{v}\left(t_{v}^{\prime}-t_{v}\right)<1 / m$ the condition $\sum_{v}\left|x\left(t_{v}^{\prime}\right)-x\left(t_{v}\right)\right| \leqq 1 / n$ is satisfied, constitute a closed set in the space $C$ normed with the norm of $L_{p}$.

In connection with the proof that $R$ is closed in space $M$ it may be advantageous to note this fact:

(iii) If $x(t)$ is an element of class $M$ with the property that $\operatorname{ess} \lim _{t \rightarrow t_{1}} x(t)$ exists for almost all $t_{1} \varepsilon[0,1]$, there exists a function $y(t)$ in class $R$ which equals $x(t)$ almost everywhere.

A considerable number of the listed results can be derived at once from others by means of the following simple observation concerning relativization:

(iv) If $S$ is a metric space, $A \subset B \subset S$, and $A$ a Borel set of a certain type relative to $S$, then $A$ is a Borel set of the same type relative to the space $B$ metrized with any metric in which convergence implies convergence in the metric of $S$.

In conclusion, we should like to emphasize that the range of applicability of the methods mainly employed above is by no means restricted to Banach spaces. By way of illustration, let us consider the set $A C$ in the space $B V$ metrized with the distance function mentioned in the second paragraph of $\S 3$, which is not a Banach space. Adams and Morse (loc. cit., p. 201) have observed that $A C$ is no $G_{\delta}$ relative to $C B V \subset B V$; that it is no $G_{\delta}$ in $B V$ follows at once; that it is no $G_{\delta \sigma}$ in $B V$ may be shown easily as follows, although Lemma 4 (Banach and Mazur) is obviously not applicable. From Lemma 6 one may infer that the limit function $x(t)$ determined in the proof of Lemma 2 satisfies the condition $T_{0}^{1}(x) \leqq \lim \inf _{n \rightarrow \infty} T_{0}^{1}\left(x_{n}\right)$; in the course of that proof one may clearly impose an additional condition on the rapidity of convergence of $\left\|x_{n}-x_{n+1}\right\|_{c}$ to zero to insure $T_{0}{ }^{1}(x)>T_{0}{ }^{1}\left(x_{k}\right)-1 / 2^{k}$ for every $k$; then $x_{n}$ tends to $x_{\varepsilon} C B V-A C$ in the metric of $B V$. The reasoning contained in the first paragraph following the proof of Lemma 2 may now be duplicated to show that $A C$ is no $G_{\delta \sigma}$ in $B V$. That $A C$ is an $F_{\sigma \delta}$ in $B V$ is an immediate consequence of $I$ and observation (iv). Since $C B V$ is already known to be a $G_{\delta}$ in $B V$, it follows at once that $A C$ is an $F_{\sigma \delta}$ but no $G_{\delta \sigma}$ relative to $C B V \subset B V$.

BROWN UNIVERSTTY,

Providence, R. I.

The University of Pennsylvania,

Philadelphi, Pa. 